\section{Update on a previously reported male with a FLNA missense mutation}

European Journal of Human Genetics (2017) 25, 905-906; doi:10.1038/ejhg.2016.156; published online 4 January 2017

We read with interest the article by Oda et al ${ }^{1}$ expanding the phenotypic and mutation spectrum of FLNA mutations in surviving males. In this regard, we would like to provide an update on a now adult male with an FLNA variant who was originally reported in this journal by Reinstein et al (Case M3) in 2013 but whose clinical phenotype has evolved significantly since publication.

At the time of original report, Case M3 was 16 years. He had been diagnosed clinically with a connective tissue disorder - Ehlers Danlos syndrome - in the neonatal period after he was noted to have soft skin with increased elasticity and hypotonia. He was subsequently found to have diaphragmatic eventration and when on to develop spontaneous pneumothoraces, joint hypermobility and mild aortic root dilatation. ${ }^{2}$ At 14 years, a MRI brain to investigate a history of headaches demonstrated bilateral periventricular nodular heterotopia. Sequencing of the FLNA locus revealed a novel maternally inherited hypomorphic missense variant, (NM_001456.1:c.381G > C, NP_001447.2 p.(Lys127Asn)). This is listed on the gene variant database at www.lovd.nl/FLNA (individual ID 00074612). His mother had mild joint hypermobility (Beighton hypermobility score 4/9). He was intellectually normal and attending mainstream school, with no history of seizures. He has no dysmorphic or bone features suggestive of an OPD spectrum disorder.

Since then, at age of 17, he required gastroplexy for gastric volvulus and malrotation on a background of recurrent abdominal pain and vomiting likely secondary to small bowel obstruction. During the laparotomy, he was found to have a 'wandering spleen' and a stretched gastrocolic omentum with multiple openings that were facilitating internal hernias. These were managed surgically with excision of the greater omentum and splenic tethering. Following the operation, he developed incisional hernias that required surgical repair. He also had surgery for a left-sided testicular torsion and had a right-sided orchidopexy at the age of 19.

Recently aged 20, he has required a prolonged hospital admission of 5 months. He presented initially with a right-sided pneumothorax, and then developed a recurrent mid-gut volvulus. He has underwent three laparotomies including a bowel resection and eventually a total colectomy with stoma formation. He currently requires nasojejunal feeding having previously had a period of time on total parenteral nutrition. He developed marked splenomegaly and portal hypertension with associated gastric varices secondary to splenic vein thrombosis, which ultimately led to a splenectomy. He has been admitted to intensive care on three occasions. This hospital admission occurred on a background of a 4 year history of severe chronic bilateral flank pain of unknown origin for which he attends a chronic pain clinic. As a result of the burden of his illness and hospital commitments, he left school at age 17 and completed year 11 and year 12 English by distance education.

Males with $\mathrm{PNH}$-associated FLNA variants who survive past infancy are rare with $<30$ cases described in the literature and even fewer reported cases surviving into adulthood. The intestinal manifestations in our patient are particularly marked and based on the paper by Kapur et al may relate to an underlying myopathic process. ${ }^{3}$ The features seen in our patient including his wandering spleen, recurrent bowel obstruction and hernias have been previously reported individually in other patients. ${ }^{1-5}$ However to our knowledge, this is the first reported case of these features developing later in life after a relatively stable neonatal period and childhood.

The majority of patients with FLNA-associated PVNH are females with most variants that affect function being lethal in males. ${ }^{5}$ Surviving males usually have missense or distal truncating variants that are associated with some residual protein function ${ }^{6-9}$ and this extends to males with other FLNA-related phenotypes. ${ }^{5}$ Hehr et al described a male with splice site mutation in FLNA who had PVNH, severe constipation and facial dysmorphism. ${ }^{9}$ They showed that the splice site resulted in both normal and aberrant mRNA transcripts and they attributed his non-lethal phenotype to him retaining some normal Filamin A function. ${ }^{9}$ Oda et al proposed that in-frame exome skipping of a $4 \mathrm{bp}$ deletion in FLNA in two boys was associated with a milder phenotype. Our patient has a missense variant in the $\mathrm{CH} 1$ domain of FLNA, where the majority of PVNH variants are reported, and is predicted to interfere with the actin binding site. ${ }^{2}$ This update on our patient provides further insight into the phenotype of males with PVNH and FLNA variants that affect protein function and demonstrates that even those that appear mildly affected in early childhood and adolescence should be actively monitored for complications later in life. It also highlights the psychological and physical burden of this condition on the patient, and difficulty in counselling and managing patients with novel FLNA missense variants.

\section{CONFLICT OF INTEREST}

The author declares no conflict of interest.

\section{ACKNOWLEDGEMENTS}

We thank the patient and his family for their commitment to disseminate the information about this rare condition.

Maie Walsh ${ }^{\star, 1}$, Geoffrey Hebbard ${ }^{2,3}$ and Alison Trainer ${ }^{1}$ ${ }^{1}$ Adult Genetic Medicine, Royal Melbourne Hospital, Melbourne, VIC, Australia;

${ }^{2}$ Department of Gastroenterology and Hepatology, Royal Melbourne Hospital, Melbourne, VIC, Australia;

${ }^{3}$ Department of Medicine, University of Melbourne, Melbourne, VIC, Australia

E-mail:maie.walsh@mh.org.au

1 Oda H, Sato T, Kunishima S et al: Exon skipping causes atypical phenotypes associated with a loss-of-function mutation in FLNA by restoring its protein function. Eur J Hum Genet 2016; 24: 408-414.

2 Reinstein E, Frentz S, Morgan T et al: Vascular and connective tissue anomalies associated with X-linked periventricular heterotopia due to mutations in Filamin A. Eur J Hum Genet 2013; 21: 494-502. 
3 Kapur RP, Robertson SP, Hannibal MC et al. Diffuse abnormal layering of small intestinal smooth muscle is present in patients with FLNA mutations and $x$-linked intestinal pseudo-obstruction. Am J Surg Pathol 2010; 34: 1528-1543.

4 Oegema R, Hulst JM, Theuns-Valks SD: Novel no-stop FLNA mutation causes multiorgan involvement in males. Am J Med Genet A 2013; 161a: 2376-2384.

5 Unger S, Mainberger A, Spitz C: Filamin A mutation is one cause of FG syndrome. Am J Med Genet A 2007; 143a: 1876-1879.

6 Parrini E, Ramazzotti A, Dobyns WB: Periventricular heterotopia: phenotypic heterogeneity and correlation with Filamin A mutations. Brain 2006; 129: 1892-1906.
7 Sheen VL, Dixon PH, Fox JW: Mutations in the X-linked filamin 1 gene cause periventricular nodular heterotopia in males as well as in females. Hum Mol Genet 2001; 10: 1775-1783.

8 Guerrini R, Mei D, Sisodiya S: Germline and mosaic mutations of FLN1 in men with periventricular heterotopia. Neurology 2004; 63: 51-56.

9 Hehr U, Hehr A, Uyanik G, Phelan E, Winkler J, Reardon W: A filamin A splice mutation resulting in a syndrome of facial dysmorphism, periventricular nodular heterotopia, and severe constipation reminiscent of cerebro-fronto-facial syndrome. J Med Genet 2006; 43: 541-544. 